\title{
COVID-19 \& an NGO and university developed interactive portal: a perspective from Iran
}

\author{
Elham Maserat $^{1} \cdot$ Fereshteh Jafari $^{2} \cdot$ Zeinab Mohammadzadeh $^{3}$ (D) $\cdot$ Mahasti Alizadeh $^{4} \cdot$ Anna Torkamannia $^{5}$
}

Received: 29 April 2020 / Accepted: 30 July 2020 / Published online: 17 August 2020

(C) IUPESM and Springer-Verlag GmbH Germany, part of Springer Nature 2020

\begin{abstract}
On 19 February 2020, Iran reported the initial cases of novel coronavirus (2019-nCoV). As of 21 March 2020, Iran had reported 175,927 COVID-19 cases, including 8425 deaths. One of the best approaches for responding to COVID-19 is rapid detection, early isolation, and quick treatment of the disease. Studies have stated that information technology (IT) is a powerful tool for detecting, tracking, and responding to pandemic diseases. Despite the importance of IT, a lack of efficient use of information technology capacity was observed after the emergence of the new cases of COVID-19 in Iran. A web-portal can integrate different services and technologies and can support interaction between non-governmental organizations (NGO) and universities. NGOs can provide services for public health utilizing technology and its advancements. One of the important duties of these organizations is to inform and provide integrated services to the general public. An interactive portal is one of the advanced technologies that these organizations can use for health management. Medical sciences of universities play a vital surveillance role for enhancing the performance quality of NGOs. A web-portal can be a collaboration tool between health-related NGOs and medical sciences of universities. In this study, an interactive portal was developed by NGOs and a university. NGOs under the supervision and participation of Tabriz University of Medical Sciences' Center for Social Factors Research in COVID-19 management division of this portal separated classified information into two sections, informatics and services. This portal is accessible to the general public, patients, service providers, and, importantly, policymakers and presents educational and medical research information to all users. For patients and the general public in high-risk environments, increasing information security, reducing confusion regarding finding needed information, and facilitating communication are only part of the portal's benefit. It seems that web-portal capacity is needed to control COVID-19 in the digital age. The collaboration of academic and university bodies in the context of health portals can play key roles for coverage of the COVID-19 pandemic.
\end{abstract}

Keywords COVID-19 $\cdot$ Information technology $\cdot$ Portal $\cdot$ NGO $\cdot$ University

This article is part of the COVID-19 Health Technology: Design, Regulation, Management, Assessment

Zeinab Mohammadzadeh

z.mohammadzadeh20@gmail.com

Elham Maserat

elhammaserat@gmail.com

Fereshteh Jafari

jafari.rcgld@gmail.com

Mahasti Alizadeh

alizadehm@tbzmed.ac.ir

Anna Torkamannia

annatorkmannia@gmail.com
1 Department of Medical Informatics, Faculty of Medical Science, Tarbiat Modares University, Tehran, Iran

2 Biomedical \& Environmental Health, Leicester School of Pharmacy, Health and Life Sciences Faculty, DMU, The Gateway, Leicester LE1 9BH, UK

3 Health Information Technology Department, School of Management \& Medical informatics, Tabriz University of Medical Sciences, Tabriz, Iran

4 Social Determinants of Health Research Center, Department of Community and Family Medicine, Tabriz University of Medical Sciences, Tabriz, Iran

5 Student Research Committee, School of Management \& Medical informatics, Tabriz University of Medical Sciences, Tabriz, Iran 


\section{Introduction}

Coronavirus disease 2019 (COVID-19) has quickly spread globally [1]. The novel coronavirus outbreak, which began in Wuhan, China, in December 2019, has expanded worldwide [2]. The first confirmed case of coronavirus infection in Iran occurred on 19 February 2020 [3, 4]. One of the most effective strategies for responding to COVID-19 is rapid identification, early isolation, and quick treatment of the disease [5]. Information technology (IT) can be used in various dimensions to manage COVID-19, and IT tools can facilitate prevention, screening, diagnosis, treatment, and follow-up steps. Telemedicine, as one of the new technologies being used during epidemic conditions, has high potential to improve disease control without geographic or location restrictions [6]. Expert systems can be employed to predict disease and risk assessment based on geographic area [7]. Studies have stated that IT was a powerful tool for detecting, tracking, and responding to the 2009 pandemic of influenza A (H1N1) virus $[8,9]$.

Despite the importance of these systems, a lack of efficient use of information technology capacity was observed after the emergence of the new cases of COVID-19 in Iran [10]. A web-portal can integrate different services and technologies for patients [11]. One of the critical technologies available is a web-portal that combines technologies into one platform, enabling patients to view electronic health record data, to exchange safe messages with physicians, request visits, and request prescriptions refills if needed $[12,13]$. Web-portals are increasingly a part of modern life. Portals receive data from multiple sources seamlessly and help organizations and institutions look at and integrate various applications, software, content, and information from databases [14]. Therefore, users are presented with a web page that provides them with information regarding different servers and/or systems. Portal content is accessible from a variety of devices such as PCs and smartphones $[14,15]$. Numerous studies have verified the usefulness of portals in the management of chronic diseases, and it is manifest that the portal be designed to meet the needs of users [16]. In Iran, the lack of efficient use of information technology capacity in public opinion management is considered to have caused fear and other emotional reactions in society [10]. Therefore, the need to integrate these technologies into a comprehensive portal is increasingly felt due to the numerous coherent information management systems related to the disease. Thus, considering the benefits of the health portal and its critical role in information interaction and the lack of electronic context for the communication of the various tools that have been provided to manage and monitor COVID-19, we offered this platform in the interactive portal of non-governmental organizations (NGOs), research institutes, and universities.

\section{Methods}

For the first step of the study, we performed a comprehensive search for items used to design core dimensions of an interactive portal. In this step, all relevant books, articles, research projects, theses, manuals, and scientific reports were extracted from MEDLINE, IEEE, Scholar, Web of Sciences, Scopus, Proquest, websites, and databases related to interactive portals related to health. Some of the highest priorities for designing portals extracted from scientific databases were:

- Interaction of NGOs with each other

- Ability to upload information regarding each nongovernmental organization separately

- Easy content management by users

Users' comments regarding the system should be considered before design requirements of the system model are formulated [17]. Prioritization of requirements for designing is based on users' opinions $[18,19]$.

The second step of the study was a qualitative survey. In-depth and semi-structured interviews and focus group discussions were conducted based on the views of 25 staffs of non-governmental organizations who worked on health care plans. Participants were informed about the interactive web-portal. The researcher explained the purpose of the study. In addition, researchers requested consent to audio-record the interviews. Researchers explained the study and obtained initial consent for further contact from participants. Interviewing involves asking questions and getting answers from participants. In the next step, focus groups with semi-structured discussions were used to analyze and approve the final conceptual models, content structure, and architecture of the portal. Table 1 illustrates the participants of this study. NGO participants in this study were active in five areas: education, treatment, prevention, empowerment, and health.

Various studies have been conducted to develop the system, based on the needs of users, and open source platforms have been selected due to their high availability and security [19]. In addition, PHP language, MySQL database, content management system of Joomla, HTML, and CSS were used for development. The Joomla management system creates a strong security environment and has high flexibility in user management, support, and classification [20]. Another reason for choosing this content management system is the existence of various and comprehensive plugins and providing the possiblity to develop other features in the future. In the designed system, user authentication processes, user approval, group assignments, and user roles are easily possible. 
Table 1 Participants according to educational level

\begin{tabular}{llllll}
\hline $\begin{array}{l}\text { Educational level } \\
\text { NGOs staff }\end{array}$ & Diploma & Bachelor & Master & Doctorate & Total \\
\hline Chief executive officer & $2(8 \%)$ & $8(32 \%)$ & $3(12 \%)$ & $1(4 \%)$ & $14(56 \%)$ \\
Chairman of the board & 0 & $2(8 \%)$ & 0 & 0 & $2(8 \%)$ \\
Member of the board & 0 & 0 & $1(4 \%)$ & 0 & $1(4 \%)$ \\
Chief communications officer & 0 & $4(16 \%)$ & 0 & 0 & $4(16 \%)$ \\
Financial affairs manager & 0 & $2(8 \%)$ & 0 & 0 & $2(8 \%)$ \\
Deputy manager & 0 & 0 & $1(4 \%)$ & 0 & $1(4 \%)$ \\
Secretary of the organization & 0 & 0 & 0 & $1(4 \%)$ & $1(4 \%)$ \\
Total & $2(8 \%)$ & $16(64 \%)$ & $5(20 \%)$ & $2(8 \%)$ & $25(100 \%)$ \\
\hline
\end{tabular}

\section{Results}

The interactive portal developed by the NGOs and university provides a platform for unity and integrity to function and prevents the overlap of the activities of NGOs and enables oversight by scientific bodies. NGOs, under the supervision of and in participation with Tabriz University of Medical Sciences' Center for Social Factors Research in COVID-19 Management Division of this portal, separated classified information into two sections of informatics and services. The informatics section provides protocols and guidelines for prevention, diagnostics, and treatment approved by universities and academic institutions. This NGOs pathway is formulated as an operational plan to monitor the step-by-step activities of the portal, which will be well presented to the public in a very organized way. The process of NGO's work and guidance on this portal regarding COVID-19 provides a valuable facility to the general public, in which they will be able to place their questions and receive answers. This technology has flawlessly accelerated and facilitated the responsibility of NGOs.

In the Portal Services section, the information content of NGOs services was presented in three essential phases: prevention of, treatment of, and recovery from COVID-19 (this phase included rehabilitation and death separately).

In the first section, comprehensive information regarding the process of public training, the epidemic of the disease, and follow-up are the methods provided. Additionally, step-bystep information regarding the process of tracking and protecting vulnerable, unprotected people, the elderly and Slumdog, which can be covered by some NGOs, is presented. Furthermore, the number and type of donation items such as masks, gloves, and disinfectants are available on this portal.

In the treatment section, NGOs provided adequate information regarding equipment, consumables, and the budget/costs for this disease separately, which is based on the evaluation provided by professional academic bodies. Additionally, specialist counselling is accessible to the general public and individuals with a high risk of infection. Also, shared experiences by recovered patients are available.

In the recovery area, the details of companies/communities that are ready to offer free of charge services or discount are introduced.

Furthermore, the valuable and evidence-based links regarding the information content of social networks and the national systems such as self-assessment of suspected patients, COVID-19 disease pathway detection, urban and rural high-risk system, self-care system, online counselling system, and Iranian epidemiological administration system are visually usable for both the general public and medical staff.

System performance requirements of this portal are identified as follows:

- Personalization: This system must specify the type of user access by the administrator

- Customization: The user must be able to configure how the information is displayed. For example, screen control

- Grouping: Beneficiary groups have privacy if they have group communication.

- Easy to use: Ability to access the system in different and standard browsers and display information in different tools

- Detail management: Each group should have its own management and in addition to one person have several content managers.

Principle features of active (authorized) users of portal include:

- User discussion: Ability to discuss information and interact between users based on a specific topic and focused on a specific environment

- Event Calendar: Record information about future events

- Links: Links to other web addresses that contain users' favorite information 
- Subject lists: A hierarchy of sub-lists/folders with documents that can only be accessed by active (authorized) users.

The architecture of the web-portal is presented in Fig. 1. The interactive portal developed by the NGOs and university is accessible to the general public, patients, service providers, and, importantly, policymakers and presents educational and medical research information to all users. Moreover, collecting data from various sources, making information available to all types of user groups, and providing the ability that users are capable of uploading data to the portal by themselves are some portal features. This ability would occur according to the level of access, defining personal pages by users or groups, and reducing outpatients' appointments and/or in-person visits. Identifying patients and the general public in high-risk environments, increasing information security, reducing confusion regarding finding needed information, and facilitating communication are only part of the portal's benefit (Fig. 2).

An interactive portal elaborated with the continued support of social determinants of the Health Research Center of Tabriz University of Medical Sciences and collaboration of health-related non-government organizations has been developed. In this portal, NGOs and the university share everyday experiences and activities in the field of citizen empowerment, government sectors, and citizen advocacy.

\section{Discussion}

We describe an interactive portal developed by NGOs and a university that provides a platform for unity and integrity to help manage the COVID-19 pandemic and its oversight by scientific bodies. The COVID-19 response requires new information technology approaches to support clinical needs [21]. A web-based portal related to health provides patients easier access to their healthcare information and services [11]. Our web-portal divided data into two sections, informatics and services. Consideration of informatics needs and useful guidance are vital options to pandemic response [22, 23]. In addition, media partnerships can prevent societal fear and help manage COVID-19 in low- and middle-income countries [24]. The informatics section of the interactive portal presents protocols and guidelines for prevention, diagnosis, and treatment. These protocols are approved by universities and academic institutions. The general public are monitoring the activities of NGOs in the field of COVID-19 and are asking for their help.

One of the big challenges of COVID-19 response is the health information required to safely care for a patient. Patients may not be able to select the health care provider for follow up of COVID-19 [25]. The process of NGO's work and guidance on our portal regarding COVID-19 provides a valuable facility to the general public, in which they will be able to place their questions and receive answers.

Studies have illustrated public health interventions can improve control of COVID-19, such as in Wuhan, China [26]. A
Fig. 1 Architecture of the webportal
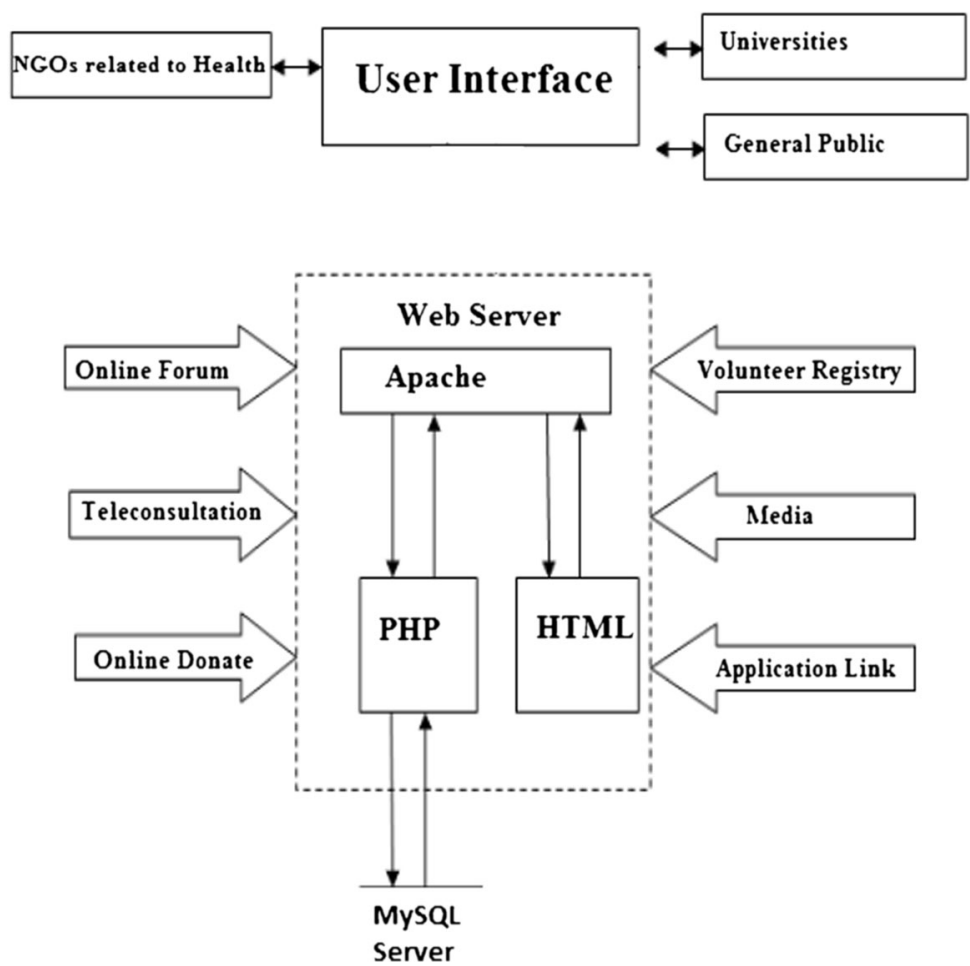


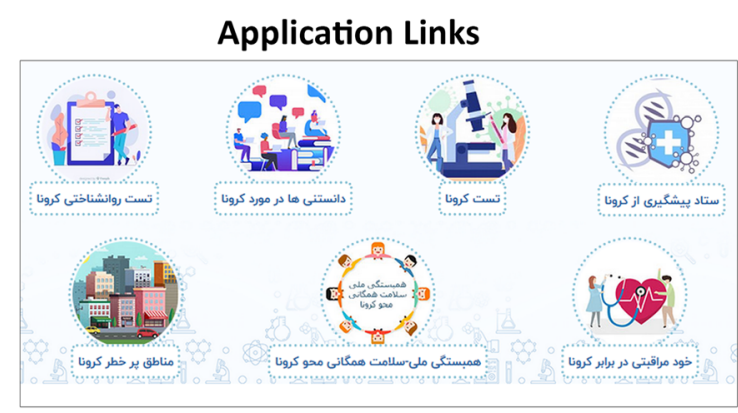

NGOs Network

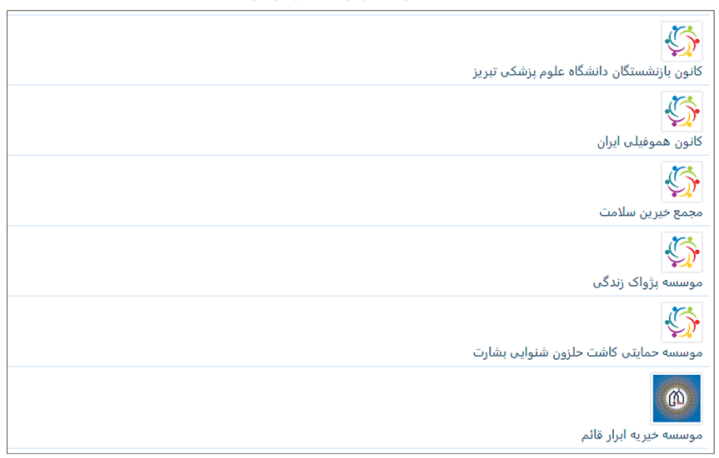

Fig. 2 Services of the web-portal

web-based portal facilitates dissemination of public health information [27], as is the case in the current study. Comprehensive monitoring is needed to notice changes in the pandemic and the effectiveness of public health interventions and their social acceptance [28]. Portals enhance communication and social acceptance. The prevention section of the web-portal will address close information regarding the process of public training, the epidemic of the disease, and follow-up the method provided. In addition, step-by-step information regarding the process of tracking and protecting vulnerable, unprotected people, the elderly and Slumdog, which can be covered by some NGOs, is presented. NGOs activities in web-portal framework are monitored by Tabriz University of Medical Sciences. Furthermore, the number and type of donation items such as masks, gloves, and disinfectants are available on this portal.

Information technology can facilitate accurate health care resource allocation in high-, low-, and middle-income countries. Electronic data collection of patients at a population level is one of the models that shows the capabilities of IT for resource allocation. We can overcome the challenges of traditional data collection using IT tools [29]. In the treatment section of the portal, NGOs provided adequate information regarding equipment, consumables, and the budget/costs for this disease separately, which is based on the evaluation provided by professional academic bodies.

Furthermore, information technology-based tools can facilitate accountability and transparency in governmental and non-governmental organizations in a country [30]. The portal
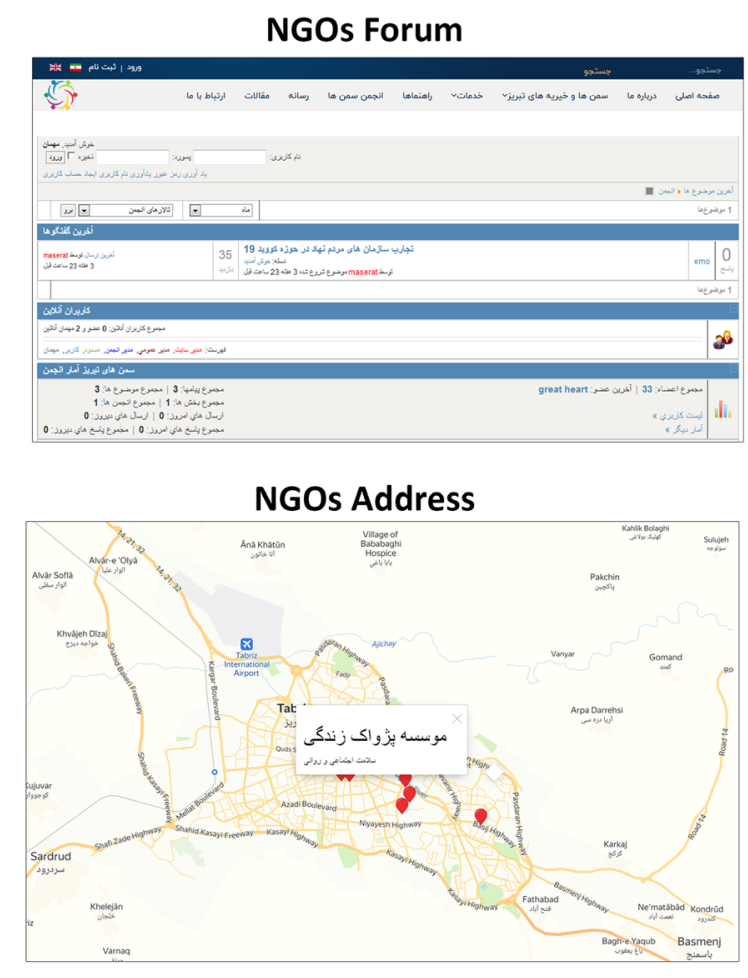

developed by the NGOs and university can support accountability and transparency in governmental and nongovernmental organizations.

\section{Conclusion}

A web-portal is an essential tool in supporting the general public needs of a health system managing the COVID-19 pandemic. The collaboration of academic and university bodies in the context of health portals brings together the most upto-date technologies that can play a significant role in attracting public sectors and crisis management.

Acknowledgements The authors thank all of the Social Determinants of Health Research Center staff and professionals involved in this study for their cooperation and support.

\section{Compliance with ethical standards}

Conflict of interest The authors declare that they have no conflict of interest.

\section{References}

1. Li Q, Guan X, Wu P, Wang X, Zhou L, Tong Y, et al. Early transmission dynamics in Wuhan, China, of novel coronavirusinfected pneumonia. N Engl J Med. 2020;382(13):1199-1207. 
2. Chinazzi M, Davis JT, Ajelli M, Gioannini C, Litvinova M, Merler S, et al. The effect of travel restrictions on the spread of the 2019 novel coronavirus (COVID-19) outbreak. Science. 2020;368(6489):395-400.

3. Takian A, Raoofi A, Kazempour-Ardebili S. COVID-19 battle during the toughest sanctions against Iran. Lancet. 2020;395(10229): 1035-1036.

4. Tuite AR, Bogoch I, Sherbo R, Watts A, Fisman DN, Khan K. Estimation of COVID-2019 burden and potential for international dissemination of infection from Iran. medRxiv. 2020; https:// doi.org/10.1101/2020.02.24.20027375

5. Wang F-S, Zhang C. What to do next to control the 2019-nCoV epidemic? Lancet. 2020;395(10222):391-3.

6. Zhai Y, Wang Y, Zhang M, Gittell JH, Jiang S, Chen B, et al. From isolation to coordination: how can telemedicine help combat the COVID-19 outbreak? medRxiv. 2020; https://doi.org/10.1101/ 2020.02.20.20025957

7. Sandhu R, Sood SK, Kaur G. An intelligent system for predicting and preventing MERS-CoV infection outbreak. J Supercomput. 2016;72(8):3033-56.

8. Brownstein JS, Freifeld CC, Chan EH, Keller M, Sonricker AL, Mekaru SR, et al. Information technology and global surveillance of cases of 2009 H1N1 influenza. N Engl J Med. 2010;362(18): 1731-5.

9. Brownstein JS, Freifeld CC, Madoff LC. Influenza A (H1N1) virus, 2009—online monitoring. N Engl J Med. 2009;360(21):2156.

10. Doshmangir L, Mahbub Ahari A, Qolipour K, Azami-Aghdash S, Kalankesh L, Doshmangir P, et al. East Asia's strategies for effective response to COVID-19: lessons learned for Iran. Management Strategies in Health System. 2020;4(4):370-3.

11. Lu S, Hong Y, Liu Q, Wang L, Dssouli R. Implementing webbased e-health portal systems. Department of Computer Science and CIISE, Concordia University. 2017.

12. Ancker JS, Barrón Y, Rockoff ML, Hauser D, Pichardo M, Szerencsy A, et al. Use of an electronic patient portal among disadvantaged populations. J Gen Intern Med. 2011;26(10):1117-23.

13. Haddad M, Chetty G, editors. Development of a smart e-health portal for chronic disease management. International conference on algorithms and architectures for parallel processing. ICA3PP 2012. Lecture Notes in Computer Science, vol 7440. Berlin: Springer; 2012.

14. Sullivan D. Proven portals: best practices for planning, designing, and developing enterprise portals. Boston: Addison-Wesley; 2003.

15. Irizarry $\mathrm{T}$, Dabbs $\mathrm{AD}$, Curran $\mathrm{CR}$. Patient portals and patient engagement: a state of the science review. J Med Internet Res. 2015;17(6):e148.

16. Wickramasinghe N. Delivering superior health and wellness management with IoT and analytics. Cham: Springer; 2020.
17. Lala O, Akinpelu J, Emuoyibofarhe O. Framework for a web based information dissemination and academic support educational portal. Proceedings of ICOMeS; 2012. p. 66.

18. Kurangi CR, Jayakumar S. An effective educational portal for visually impaired persons in India. Int J Pure Appl Math 2017;117(19):259-267.

19. Dervos DA, Psarras K, editors. A framework for the design of an extensible modular academic web site. Proceedings of the 2 nd international scientific conference on information technology and quality. Spetses, Greece; 2005.

20. Smyrnova-Trybulska E, Stach S, Staniek D. Some theoretical and practical aspects of educational portal design based on CMS system. 2012; http://hdl.handle.net/20.500.12128/7928

21. Grange ES, Neil EJ, Stoffel M, Singh AP, Tseng E, RescoSummers K, et al. Responding to COVID-19: the UW medicine information technology services experience. Appl Clin Inform. 2020;11(02):265-75.

22. Fidler DP, Gostin LO. The WHO pandemic influenza preparedness framework: a milestone in global governance for health. Jama. 2011;306(2):200-1.

23. Organization WH. Pandemic influenza preparedness and response: a WHO guidance document: Geneva: World Health Organization; 2009.

24. Hopman J, Allegranzi B, Mehtar S. Managing COVID-19 in lowand middle-income countries. Jama. 2020;323(16):1549-50.

25. Lenert L, McSwain BY. Balancing health privacy, health information exchange, and research in the context of the COVID-19 pandemic. J Am Med Inform Assoc. 2020;27(6):963-6.

26. Pan A, Liu L, Wang C, Guo H, Hao X, Wang Q, et al. Association of public health interventions with the epidemiology of the COVID-19 outbreak in Wuhan, China. Jama. 2020;323(19):1-9.

27. Murray M, editor An investigation of specifications for migrating to a web portal framework for the dissemination of health information within a public health network. Proceedings of the 35th Annual Hawaii International Conference on System Sciences. IEEE. 2002.

28. Heymann DL, Shindo N. COVID-19: what is next for public health? Lancet. 2020;395(10224):542-5.

29. Arnold M, Soerjomataram I. Global chemotherapy demands: a prelude to equal access. Lancet Oncol. 2019;20(6):742-3.

30. Sofyani H, Riyadh HA, Fahlevi H. Improving service quality, accountability and transparency of local government: the intervening role of information technology governance. Cogent Bus Manag. 2020;7(1):1735690.

Publisher's note Springer Nature remains neutral with regard to jurisdictional claims in published maps and institutional affiliations. 